\title{
Subcutaneous Allergen Immunotherapy in Children: Real Life Compliance and Effect of COVID-19 Pandemic on Compliance
}

\author{
Elif Soyak Aytekin Özge Soyer Bülent E. Şekerel Ümit M. Şahiner \\ Department of Pediatric Allergy, Hacettepe University School of Medicine, Ankara, Turkey
}

\section{Keywords}

Subcutaneous allergen immunotherapy · Children .

Compliance · Coronavirus disease 2019

\begin{abstract}
Background: Subcutaneous allergen immunotherapy (SCIT) is an effective treatment for allergic rhinitis, asthma, and venom allergy. Compliance is essential for SCIT to obtain maximal benefit as it is a long-term treatment. Objectives: This study aimed to determine the level of real-life SCIT compliance in pediatric patients and the associated factors. Additional aims were to determine how SCIT compliance was affected by the COVID-19 pandemic and why some patients dropped out SCIT. Method: Pediatric patients diagnosed with allergic rhinitis, allergic asthma, or venom allergy that received SCIT between September 2012 and July 2020 were analyzed. Results: The study included 201 children ( $66.7 \%$ male) with a median (interquartile range) age of 12.8 years (9.4-15.2) at the time of the first SCIT injection. The overall compliance rate before COVID-19 pandemic was $86.1 \%$. Short SCIT follow-up time and venom anaphylaxis were found to be risk factors for drop out. The leading causes of drop outs were moving to another city/ country (32.1\%), symptom improvement (17.8\%), treatment ineffectiveness (14.2\%), and adverse reactions (14.2\%). Among the 108 patients that were still receiving SCIT during
\end{abstract} The most frequent reasons for drop-out were fear of being infected with COVID-19 (35.4\%) and thinking that the AIT practise stopped due to COVID-19 pandemic (29\%). Male gender and older age were found to be the independent risk factors for drop-out of SCIT. Conclusions: Real life compliance in children was found $13.9 \%$ and it was higher than adults. Nearly one-third of children dropped out during the COVID-19 pandemic. Male gender and older age are associated with SCIT drop-out during the COVID-19 pandemic.

(c) 2021 S. Karger AG, Basel

\section{Introduction}

Allergen immunotherapy (AIT) is an effective and game-changing treatment method for allergic rhinitis (AR), venom anaphylaxis, and allergic asthma that can provide immune tolerance for many years. Subcutaneous allergen immunotherapy (SCIT) is the most commonly used administration route, and requires repeated administration of allergen extracts for 3-5 years, depending on the type of allergen administered.

Edited by: H.-U. Simon, Bern. 
Treatment compliance is essential for SCIT to obtain maximal benefit, as it is a long term treatment; however, the non-compliance rate can be as high as $50 \%$ in both adults and children [1-3]. The major factors associated with non-compliance are long duration of treatment, frequency of injections, high cost, improvement of systemic reactions over time, poorly perceived efficacy, allergic reactions during vaccinations, and travelling, and these factors vary according to heathcare center, health system structure, and cultures [4-6]. Most studies on SCIT compliance are adult-based or population-based (both adults and children); relevant pediatric studies are limited in number [3].

The Coronavirus disease 2019 (COVID-19) pandemic not only affected people with COVID-19 infection, but also disrupted the treatment of patients with chronic diseases. During the COVID-19 pandemic the pattern of hospital admissions and presentations changed noticeably, and included a decrease in pediatric outpatient presentations for SCIT. The present study aimed to determine the level of real life compliance in pediatric patients and to determine the associated factors. Additional aims were to determine how SCIT compliance was affected by the COVID-19 pandemic and why some patients dropped out the treatment.

\section{Methods}

\section{Study Population}

The study included 201 pediatric patients with AR, allergic asthma or venom anaphylaxis and that underwent SCIT at Hacettepe University Medical School Hospital, Department of Pediatric Allergy, between September 2012 and July 2020.

SCIT was administered to patients with AR and/or allergic asthma that had uncontrolled AR symptoms, along with medical treatment and the prescription of avoidance measures. SCIT was given to patiens with asthma only if their disease was allergic and mild to moderate in severity. Venom immunotherapy (VIT) was administered to patients with a history of $\geq 1$ systemic reactions after Apis mellifera and/or Vespula vulgaris stings, and positive diagnostic test reactions (skin prick test [SPT] or specific immunoglobulin E [sIgE]) for culprit insect venom.

The treatment compliance of patients in COVID-19 period was defined as the time between the first confirmed case of COVID-19 in Turkey (March 15, 2020) and June 15, 2020. The study protocol was reviewed and approved by our University Institutional Review Board.

\section{Diagnosing AR, Allergic Asthma, and Venom Allergy}

The diagnoses and therapies of AR, asthma, and venom allergy were based on AR and its impact on asthma (ARIA) [7], Global Initiative for Asthma (GINA) [8] and European Academy of Allergy and Clinical Immunology guidelines [9], respectively.
Administration of SCIT and Compliance Assessment

Patients were each administered one of the following: Alutard SQ $100 \%$ V. vulgaris or A. mellifera (Alutard SQ, ALK, Hørsholm, Denmark) VIT, Allergovit Grass 006 (Allergopharma Joachim Ganzer KG, Hamburg, Germany) allergen immunotherapy; NovoHelisen Depot HDM 50\% DP + 50\% DF (Allergopharma Joachim Ganzer KG, Hamburg, Germany) allergen immunotherapies. VIT doses were administered at 1-week intervals and were gradually increased to the maintenance dose over the course of 6 months [10]. Then, maintenance doses were administered every 4-6 weeks for $\leq 5$ years [11]. AIT doses for grass allergy and house dust mite (HDM) allergy were administered at 1-week intervals, and were then gradually increased to the maintenance doses over the course of 6 weeks for grass and 14 weeks in HDM [12]. Then, maintenance doses were administered every 4-6 weeks for $\leq 4$ years.

Treatment compliance was defined as receiving SCIT according to the prescribed treatment course and evaluated during the 1st year, 2nd year, 3rd year, 4th year, and 5th year, or at treatment completion. Patients with $\leq 2$ two-week breaks from their prescribed treatment schedule per year were considered to have excellent compliance, 3-4 two-week breaks was considered good compliance, 5-6 two-week breaks were considered fair compliance, and $\geq 7$ two-week breaks were considered poor compliance [13]. Patients who had 3 consecutive months delay from their defined treatment schedule were accepted as dropped out [14], and the reasons for drop-out were recorded.

\section{Statistics}

Statistical analyses were performed using SPSS version 22.0 statistical software package (IBM SPSS Statistics, Philadelphia, PA, USA). First normality tests for continuous variables were performed and as all of the continuous variables were distributed nonnormally the results were given as median (inter-quartile range $[\mathrm{IQR}])$. The $\chi^{2}$ and Mann-Whitney $U$ tests were used to compare nonparametric values. Logistic regression analysis for risk assessment and Kaplan-Meier analysis for change in compliance by time were used. The level of statistical significance was set at $p<0.05$.

\section{Results}

\section{Real-Life Compliance before COVID-19 Pandemic}

(September 2012 - March 2020)

In all, 201 children who received SCIT injections between 2012 September and $2020 \mathrm{March}$. According to the demographic data, males were predominant $(134,66.7 \%)$. Median age at the start of SCIT was 12.8 years (9.4-15.2) and median treatment duration was 40.7 months (20.449.9). Patient demographic characteristics are summarized in Table 1.

In total, 154 (76.6\%), 10 (5\%) and 37 (18.4\%) patients were treated with SCIT for AR, asthma and venom anaphylaxis, respectively. Pollen immunotherapy was the most common form of SCIT (78.1\%), followed by VIT (18.4\%) and HDM (3.5\%). Among the patients, 93 (46.2\%) 
Table 1. Demographic and clinical characteristics of study population

\begin{tabular}{|c|c|c|c|c|}
\hline & $\begin{array}{l}\text { Whole group } \\
(n=201)\end{array}$ & $\begin{array}{l}\text { Compliant group } \\
(n=173)\end{array}$ & $\begin{array}{l}\text { Drop-out group } \\
(n=28)\end{array}$ & $p$ value \\
\hline SCIT start age ${ }^{\star}$ & $12.8(9.4-15.2)$ & $12.9(9.9-15.3)$ & $11.1(7.5-14.8)$ & 0.081 \\
\hline Current age* & $15.9(13.1-18.8)$ & $16.0(13.2-18.8)$ & $15.5(11.6-18.8)$ & 0.250 \\
\hline Gender M (\%) & $134(66.7)$ & $112(64.7)$ & $22(78.6)$ & 0.150 \\
\hline SCIT duration & $40.7(20.4-49.9)$ & $43.5(24.1-50.4)$ & $23.6(14.3-35.6)$ & $<0.001$ \\
\hline AR \pm Asthma, $n(\%)$ & $154(76.6)$ & $138(79.8)$ & $16(57.2)$ & \multirow{3}{*}{$<0.001$} \\
\hline Venom, $n(\%)$ & $37(18.4)$ & $31(17.9)$ & $6(21.4)$ & \\
\hline Family history of atopy, $n(\%)$ & $96(47.8)$ & $84(48.5)$ & $12(42.8)$ & \\
\hline \multicolumn{5}{|l|}{ Type of SCIT, $n(\%)$} \\
\hline Grass & $157(78.1)$ & $135(78.1)$ & $22(78.6)$ & \multirow[b]{2}{*}{0.523} \\
\hline Venom & $37(18.4)$ & $31(17.9)$ & $6(21.4)$ & \\
\hline
\end{tabular}

SCIT, subcutaneous allergen immunotherapy; AR, allergic rhinitis; M, male. * Median (interquartile range).

Table 2. Reasons of drop-outs before COVID-19 pandemics and during COVID-19 pandemic

\begin{tabular}{|c|c|c|c|}
\hline \multicolumn{2}{|c|}{$\begin{array}{l}\text { Before COVID-19 pandemic } n(\%) \\
\text { (total population: } 201 \text { ) }\end{array}$} & \multicolumn{2}{|l|}{$\begin{array}{l}\text { During COVID-19 pandemic } n(\%) \\
\text { (total population: } 108)\end{array}$} \\
\hline Systemic reaction & $4(14.3)$ & Fear of being infected with COVID-19 & $11(35.4)$ \\
\hline Moving to another city & $9(32.1)$ & The belief that SCIT practice was stopped due to COVID-19 & $9(29)$ \\
\hline Long distance & $2(7.1)$ & Problems of transportation & $7(22.7)$ \\
\hline Poor efficacy of AIT & $4(14.3)$ & Inability to access allergen extract & $2(6.5)$ \\
\hline Improvement of symptoms & $5(17.9)$ & Having a COVID-19 infected family member & $1(3.2)$ \\
\hline Frequency of injections & $3(10.7)$ & Few doses left to finalize the treatment & $1(3.2)$ \\
\hline Inability to access medication & $1(3.6)$ & & \\
\hline Total drop-outs & $28(100)$ & Total drop-outs & $31(100)$ \\
\hline
\end{tabular}

SCIT, subcutaneous allergen immunotherapy.

completed the treatment and 108 patients continued to receive SCIT during the course of the study. The overall SCIT compliance rate was $86.1 \%$ ( 173 out of 201 patients). In all, 28 patients (13.9\%) stopped AIT before recommended time; 2 patients dropped out during year 1 (0.99\%), 10 during year 2 (5.8\%), and $16(10.8 \%)$ after 2 years of treatment. Among the compliant group $(n=$ 173), all had excellent compliance during the 1st and 2 nd years of therapy. After 2 years of therapy, $93.9 \%$ of patients had excellent compliance, 4.6 and $1.5 \%$ had good and fair compliance, respectively. The change in compliance over time in the whole group; in females versus

Subcutaneous Allergen Immunotherapy

Compliance and COVID-19 Pandemic males; and in asthma, allergic rhinitis and venom anaphylaxis patients were shown in Figure 1a-c respectively.

The duration of treatment in the drop-out group was shorter $(p<\mathbf{0 . 0 0 1})$ and venom anaphylaxis and asthma were significiantly higher compared to compliant group $(p<0.001)$ (Table 1$)$. Moving to another city/country (32.1\%) was the most frequent reason for drop-out (Table 2).

\section{The COVID-19 Pandemic Period}

The COVID-19 pandemic started officially in midMarch 2020 in our country. At that time, 108 patients 


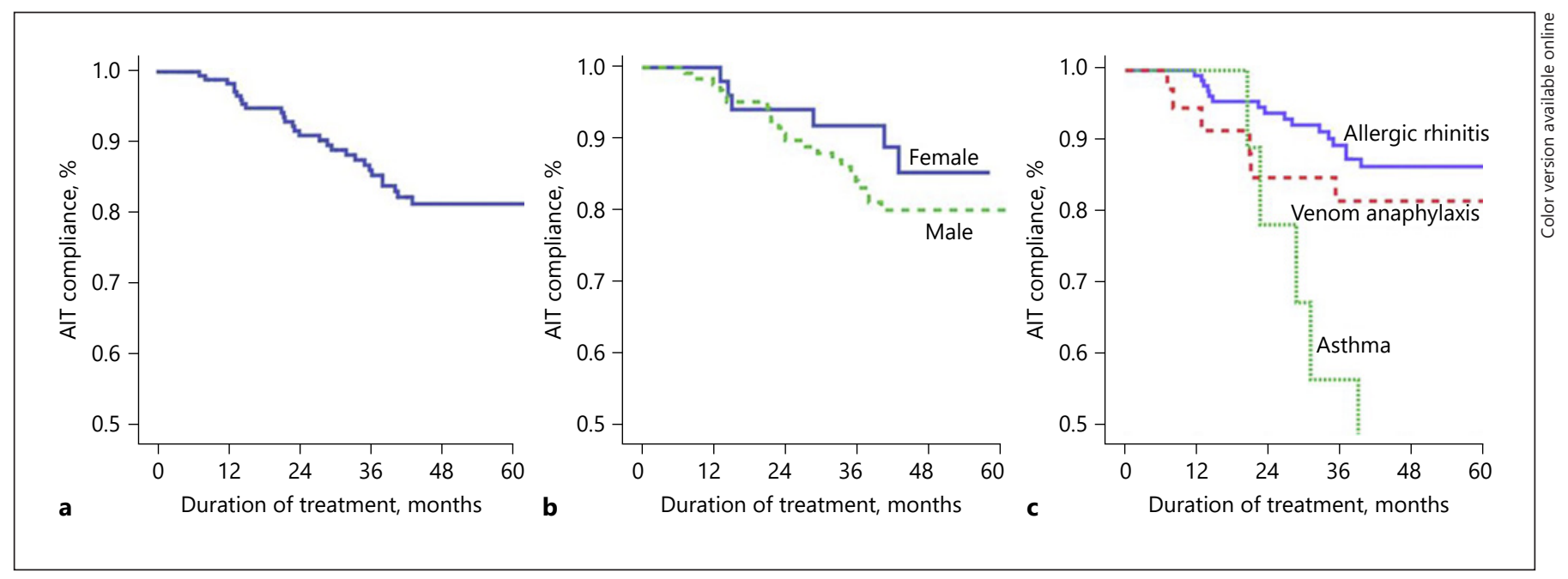

Fig. 1. Allergen immunotherapy compliance over time in the whole group (a), in male and female (b), and in allergic rhinitis, asthma and venom anaphylaxis patients (c).

were receiving SCIT, of which 31 (28.7\%) dropped out the therapy and $15(13.8 \%)$ had breaks in their treatment. Among the drop-out group, the median treatment duration was 40 months (29-49.8). The most frequent reason for drop-out was fear of being infected with COVID-19 (35.4\%), followed by the belief that the SCIT practise stopped due to the COVID-19 pandemic (29\%) (Table 2).

In the drop-out group, median age of the patients and percentage of males were higher compared to the compliant group (17.5 years [13.6-19.2] vs. 14.5 years [10.817.1], $p=0.004$ and 66.7 vs. $53.2 \%, p=0.045)$, respectively. Additionally, AIT duration was higher in the dropout group compared to the compliant group (40.0 months [29.0-49.8] vs. 28.6 [9.8-42.2] months, $p=0.018)$. We further performed univariate and multivariate logistic regression analysis for the risk factors for the development of the drop-out during the COVID-19 pandemic. Male gender (OR: 2.972, 95\% CI: 1.132-7.804, $p=0.027$ ) and higher age (OR: 1.209, 95\% CI: $1.064-1.375, p=0.004$ ) were found to be the independent risk factors for dropout during the COVID-19 pandemic.

\section{Discussion}

SCIT compliance rate ranges from 44 to $89 \%$. In the present study the compliance rate was higher than reported earlier [3]. Similar to the present findings, Pajno et al. [4] observed SCIT compliance in children of $89 \%$. Among a population of 311 allergic adult and pediatric patients receiving HDM SCIT in China, 34.5\% were noncompliant. Ineffectiveness was the leading reason for drop-out (28\%) [14]. The cost of SCIT in our country is covered by the national social security system, which might have been a major reason for high compliance rate observed in the present study.

The present findings show that SCIT drop-out was significiantly higher in the patients treated for asthma and venom anaphylaxis. As the patients that received VIT lived in a different city, most of them dropped out due to the long-distance commute and/or frequency of injections. One study reported that allergic conjunctivitis was associated with SCIT non-compliance, whereas another study reported that patients with asthma and AR were more compliant $[15,16]$. More and Hagan [17] reported that the type of respiratory allergic disease was not correlated with SCIT compliance.

Studies that compared SCIT compliance according to age have reported inconsistent findings [18, 19]. Yang et al. [14] noted that children had a higher compliance rate than adults, whereas Lee et al. [20]observed that patients aged $<20$ years and $20-40$ years were more likely to be non-compliant than those aged $>40$ years. Rhodes [21] reported that non-compliant patients were younger than compliant patients.

In the present study, gender did not affect the SCIT compliance rate before the COVID-19 pandemic; however, during the pandemic period males dropped out more than females, significantly. Musa et al. [5] and Gelincik et al. [19] found no correlation with gender, 
whereas Yang et al. [14] and Lower et al. [2] reported that SCIT compliance was higher among males.

The present findings show that moving to another city was the most common reason (32.9\%) for SCIT drop-out, followed by symptom improvement (17.9\%). Lourenco reported that $23 \%$ of patients dropped out during SCIT because of clinical improvement, primarily during the 2nd and 3rd years of treatment [18]. Yang et al. [14] reported it as $22 \%$.

We found treatment ineffectiveness (14.3\%) as another important reason of AIT drop-outs. Ineffectiveness was reported in previous studies in $8-66 \%$ of drop-out results $[1,4,14,18,19]$. Adverse reactions were also reported in several studies as a reason for drop-outs in 3.9 to $-11 \%$ of drop-outs $[18,21]$. Systemic reactions were also one of the leading reasons for drop-out (14.3\%) in the present study although it was not reported frequently in our center previously $[22,23]$. In contrast to literature, the cost of SCIT was not a reason for drop-out in the present study, most likely because it is paid for social security system of our country.

In the present study, fear of being infected with COVID-19 was the most common reason for SCIT drop-out during the COVID-19 pandemic. Although all necessary precautions were taken, the patients chose not to continue to SCIT, as the hospital was a COVID-19 pandemic center. In all, $29 \%$ of the patients dropped because they thought SCID was cancelled based on news reports that the hospital was admitting only emergencies. In addition, during the COVID-19 pandemic period, all intercity travel was banned and, in most of the cities curfews were put into effect. As a result of this situation $22.7 \%$ of patients dropped out due to transportation problems. In addition, $6.5 \%$ of the patients could not access to allergen extract and 1 patient was unable to continue SCIT, as his father (a health worker) was hospitalized with COVID-19 pneumonia. One patient's AIT was terminated earlier, who was an out of town patient and in the final months of AIT.

In the present study, older age and male gender were correlated with drop-out during the COVID-19 pandem- ic. Closure of schools and home-quarantine during pandemic was reported to cause anxiety in adolescents [24].

In conclusion the present study showed that the SCIT compliance in pediatric patients is $86.1 \%$ and higher than adults. Moving to another city, improvement of symptoms and treatment ineffectiveness are the leading reasons for drop-out. In addition, the COVID-19 pandemic significiantly reduced the SCIT compliance rate, nearly one-third of children, especially in older ages and males.

\section{Acknowledgements}

We thank to the pediatric allergy department staff that prepared, coordinated and administered the SCIT program.

\section{Statement of Ethics}

The study was conducted ethically in accordance with the World Medical Association Declaration of Helsinki, and the use of data for this study was approved by the local Ethics Committee of Hacettepe University, Ankara Turkey (IRB No. 2020/14-15) Due to the retrospective nature of the study anonymity of the data ethical comittee did not request informed consent from the study participants and families.

\section{Conflict of Interest Statement}

The authors have no conflicts of interest to declare.

\section{Funding Source}

The authors did not receive any funding.

\section{Author Contributions}

E.S.A. collected the data and wrote the article, O.S. made the statistical analysis, B.E.S. contiributed in the study design and the discussoin, U.M.S. planned the study and supervised the whole manuscript.

\section{References}

1 Cohn JR, Pizzi A. Determinants of patient compliance with allergen immunotherapy. J Allergy Clin Immunol. 1993;91(3):734-7.

2 Lower T, Henry J, Mandik L, Janosky J, Friday GA Jr. Compliance with allergen immunotherapy. Ann Allergy. 1993;70(6):480.

Subcutaneous Allergen Immunotherapy

Compliance and COVID-19 Pandemic
3 Incorvaia $\mathrm{C}$, Mauro M, Ridolo E, Puccinelli P, Liuzzo M, Scurati S, et al. Patient's compliance with allergen immunotherapy. Patient Prefer Adherence. 2008;2:247.

4 Pajno GB, Vita D, Caminiti L, Arrigo T, Lombardo F, Incorvaia $\mathrm{C}$, et al. Children's compliance with allergen immunotherapy according to administration routes. J Allergy Clin Immunol. 2005;116(6):1380.
5 Musa F, Al-Ahmad M, Arifhodzic N, Al-Herz W. Compliance with allergen immunotherapy and factors affecting compliance among patients with respiratory allergies. Hum Vaccin Immunother. 2017;13(3):514-7.

6 Cox LS, Hankin C, Lockey R. Allergy immunotherapy adherence and delivery route: location does not matter. J Allergy Clin Immunol Pract. 2014;2(2):156-60. 
7 Brożek JL, Bousquet J, Baena-Cagnani CE, Bonini S, Canonica GW, Casale TB, et al. Allergic rhinitis and its impact on asthma (ARIA) guidelines: 2010 revision. J Allergy Clin Immunol. 2010;126(3):466-76.

8 Pedersen SE, Hurd SS, Lemanske RF Jr, Becker A, Zar HJ, Sly PD, et al. Global strategy for the diagnosis and management of asthma in children 5 years and younger. Pediatr Pulmonol. 2011;46(1):1-17.

9 Bilo B, Rueff F, Mosbech H, Bonifazi F, OudeElberink J; Hypersensitivity EIGoIV. Diagnosis of hymenoptera venom allergy. Allergy. 2005;60(11):1339-49.

10 Golden DB. Insect sting allergy and venom immunotherapy: a model and a mystery. J Allergy Clin Immunol. 2005;115(3):439-48.

11 Jutel M, Agache I, Bonini S, Burks AW, Calderon $\mathrm{M}$, Canonica $\mathrm{W}$, et al. International consensus on allergy immunotherapy. J Allergy Clin Immunol. 2015;136(3):556-68.

12 Chaker AM, Al-Kadah B, Luther U, Neumann U, Wagenmann M. An accelerated dose escalation with a grass pollen allergoid is safe and well-tolerated: a randomized open label phase II trial. Clin Transl Allergy. 2015; 6(1):4-7.
13 Leader BA, Rotella M, Stillman L, DelGaudio JM, Patel ZM, Wise SK. Immunotherapy compliance: comparison of subcutaneous versus sublingual immunotherapy. Int Forum Allergy Rhinol. 2016;6(5):460-4.

14 Yang Y, Wang Y, Yang L, Wang J, Huang N, Wang $X$, et al. Risk factors and strategies in nonadherence with subcutaneous immunotherapy: a real-life study. Int Forum of Allergy Rhinol. 2018;8(11):1267-73.

15 Donahue JG, Greineder DK, Connor-Lacke L, Canning CF, Platt R. Utilization and cost of immunotherapy for allergic asthma and rhinitis. Ann Allergy Asthma Immunol. 1999; 82(4):339-47.

16 Mahesh PA, Vedanthan PK, Amrutha DH, Giridhar BH, Prabhakar AK. Factors associated with non-adherence to specific allergen immunotherapy in management of respiratory allergy. Indian J Chest Dis Allied Sci. 2010;52(2):91.

17 More DR, Hagan LL. Factors affecting compliance with allergen immunotherapy at a military medical center. Ann Allergy Asthma Immunol. 2002;88(4):391-4.

18 Lourenco T, Fernandes M, Coutinho C, Lopes A, Spinola Santos A, Neto M, et al. Subcutaneous immunotherapy with aeroallergens evaluation of adherence in real life. Eur Ann Allergy Clin Immunol. 2020;52(2):84-90.
19 Gelincik A, Demir S, Olgaç M, İşsever H, Khishigsuren B, Özşeker F, et al. High adherence to subcutaneous immunotherapy in a reallife study from a large tertiary medical center. Allergy Asthma Proc. 2017;38(6):78-84.

20 Lee J-H, Lee S-H, Ban G-Y, Ye Y-M, Nahm D-H, Park H-S, et al. Factors associated with adherence to allergen specific subcutaneous immunotherapy. Yonsei Med J. 2019;60(6): 570.

21 Rhodes BJ. Patient dropouts before completion of optimal dose, multiple allergen immunotherapy. Ann Allergy Asthma Immunol. 1999;82(3):281-6.

22 Cetinkaya PG, Esenboğa S, Soyer ÖU, Tuncer A, Şekerel BE, Şahiner ÜM. Subcutaneous venom immunotherapy in children: efficacy and safety. Ann Allergy Asthma Immunol. 2018;120(4):424-8.

23 Gur Cetinkaya P, Kahveci M, Esenboğa S, Karaatmaca B, Soyer O, Sekerel BE, et al. Systemic and large local reactions during subcutaneous grass pollen immunotherapy in children. Pediatr Allergy Immunol. 2020;31(6):643-50.

24 Kilincel SKO, Muratdagi G, Aydin A, Usta $\mathrm{MB}$. Factors affecting the anxiety levels of adolescents in home-quarantine during $\mathrm{CO}$ VID-19 pandemic in Turkey. Asia Pac Psychiatry. 2020 Aug 11;e12406. 